\title{
Global cardiac surgery: lessons learnt from the global neurosurgery movement
}

\author{
Dominique Vervoort, ${ }^{1}$ Kee B Park, JaBaris D Swain ${ }^{2}$
}

\begin{abstract}
${ }^{1}$ Program in Global Surgery and Social Change, Harvard Medical School, Boston, Massachusetts, USA

${ }^{2}$ Division of Cardiovascular Surgery, Hospital of the University of Pennsylvania, Philadelphia, Pennsylvania, USA
\end{abstract}

Correspondence to Dr Dominique Vervoort, Program in Global Surgery and Social Change, Harvard Medical School, Boston, Massachusetts 02115-6019, USA; vervoortdominique@ hotmail.com

\section{Check for updates}

(C) Author(s) (or their employer(s)) 2019. No commercial re-use. See rights and permissions. Published by BMJ.

To cite: Vervoort $D$, Park KB, Swain JBD. Heart Asia 2019;11:e011125. doi:10.1136/

heartasia-2018-011125

\section{INTRODUCTION}

Surgical care is increasingly considered as a crucial pillar of robust health systems. Five billion people worldwide lack access to safe surgical care, responsible for 16.9 million deaths every year and one-third of the global burden of disease. ${ }^{1}$ Contrary to previous belief, investing in surgical care is cost-effective on an individual and macroeconomic level, preventing a loss of 12.3 trillion dollars in economic growth by low-income and middle-income countries (LMICs) by $2030 .^{1}$

In the past decade, the field of global surgery has gradually evolved, with its zenith paralleling the 2015 launch of the Lancet Commission on Global Surgery and the WHO Resolution WHA68.15. Since this release, several surgical subspecialties have gained important ground to create awareness surrounding the importance of their respective fields within the wider discussions. For example, the World Federation of Societies of Anaesthesiologists established a global platform for anaesthesiologists focused on capacity-building and promoting safety and quality of care, particularly in resource-constrained settings. Paediatric surgery has developed a global consensus group within the Global Initiative for Children's Surgery, addressing its global disparities. Neurosurgery, once discarded as too complex for LMICs, has evolved to become a mainstay of global health discussions. Strategic efforts to heighten visibility and awareness about the need for expansion of neurosurgical services have led neurosurgery to become a major priority of the WHO's Emergency and Essential Surgical Care Programme.

Cardiac surgery, however, can be considered the unborn child of global surgery, with limited unified efforts to address the $>4.5$ billion people without access to cardiac surgery. ${ }^{2}$ To illustrate, the third edition of the Disease Control Priorities, examining the global burden of disease and cost-effective interventions, does not include any cardiac intervention in the 44 proposed essential surgical procedures, despite cardiovascular diseases (CVD) continuing to lead as major cause of mortality worldwide with 17.65 million deaths per year. ${ }^{3}$ Here, we look at the recent and rapidly evolving global neurosurgery movement in an attempt to highlight the lessons learnt and their applicability for a similar movement for global cardiac surgery.

\section{GLOBAL NEUROSURGERY}

Shortly after the passage of the WHA68.15, neurosurgeon Dr Walter Johnson was appointed as new lead of the EESC Programme at the WHO. Together with Dr Kee Park, Korean American neurosurgeon working as a World Federation of Neurosurgical Societies (WFNS) volunteer in Phnom Penh, Cambodia, and Dr Robert Dempsey, chairman of the Coordinating Committee for International Initiatives at the WFNS, an informal group was started to address the global (and grossly unmet) need for neurosurgical care. This led to the introduction of existing global inequities within academic neurosurgery published in World Neurosurgery in April 2016, calling for the need for consensus-building with inclusion of surgeons and stakeholders from developed and developing regions to address the issue. $^{4}$

Following this, input from each world region was integrated into the Bogota Declaration on Global Neurosurgery at the International Conference on Recent Advances in Neurotraumatology Meeting 2016 in Bogota, Colombia. The Declaration puts forward priorities to address the unmet need of neurosurgical care around the world, addressing prehospital care, training and education, research, equipment, innovation, and advocacy and policy.

Recently, the team at the Global Neurosurgery Initiative (GNI) of the Program in Global Surgery and Social Change (PGSSC) at Harvard Medical School reported that 5 million emergency and essential neurosurgical operations are unmet each year, requiring 23300 additional neurosurgeons. ${ }^{5}$ Further, the WFNS and PGSSC have been able to map the availability of neurosurgical facilities, workforce and essential equipment in a geospatial manner, allowing for visualisation of the areas in critical need. Accordingly, national and international policymakers can be held accountable for existing gaps in accessing neurosurgical care.

\section{GLOBAL CARDIAC SURGERY}

Ninety-three per cent of the population in LMICs lacks access to cardiac surgery, resulting in high mortality and morbidity from congenital and acquired cardiovascular disease. ${ }^{2}$ One in 100 babies is born with a CHD requiring surgery, but $<1.5 \%$ receives the surgery they need, causing 100000 babies to die every year before reaching their first birthday. ${ }^{6} 33.4$ million people live with rheumatic heart disease, for which surgical treatment for valve repair or replacement is critical; yet, $>1.2$ million people suffer fatal complications every year from untreated cardiovascular disease. ${ }^{7}$ As a result, every year, 15 million children and young adults die or become severely debilitated from their cardiac disease. $^{6}$ 
Recently, the Cape Town Declaration on Access to Cardiac Surgery in the Developing World was a first step in moving towards a global movement tackling the need for health systems strengthening to address the global burden of RHD. ${ }^{7}$ To do so, $\mathrm{CHD}$ and other CVD requiring surgery, other surgical conditions and non-surgical elements of the health system are crucial to be taken into consideration. Scaling up cardiac surgical care in a holistic manner allows for strengthening entire health systems. It is exactly this interplay the cardiac community has to create in order to move further with, and not at the cost of, other health services.

\section{LESSONS LEARNT}

Several key events were instrumental in shepherding the global neurosurgery agenda to the frontline of global surgery discussions. First, the WFNS, as a global umbrella for regional societies, was capable of uniting neurosurgeons around the globe in a progressive manner with a focus on sustainable neurosurgical programmes in LMICs. Second, the appointment of a neurosurgeon as lead of the WHO EESC partially shifted the organisational focus to neurosurgical care, including stroke and traumatic brain injury. Third, there arose an initiative to establish the advocacy organisation Global Neurosurgery, which proved vital to champion the Global Neurosurgery movement. Fourth, the consequent establishment of the GNI within the PGSSC allowed for a dynamic framework for neurosurgeons, neurosurgical residents, medical students and other researchers to serve as a leading academic team behind global neurosurgery, highlighted by the recent exponential expansion of relevant literature.

The budding global cardiac surgery community can build on the lessons learnt from global neurosurgery. First, the lack of structured integration of independent regional cardiac surgical societies within the World Society of Cardiovascular and Thoracic Surgeons limits intersocietal communication and collaborative efforts. Second, parallel with and independent from the Cape Town Declaration, the advocacy organisation Global Cardiac Surgery was established to raise awareness about the matter, providing a platform to advocate, access educational resources and highlight research in the field. Third, to date, there is no global database that documents the annual number of cardiac surgical procedures, their outcomes, the number of trained and certified cardiothoracic surgeons, or the number of qualified cardiac surgical teams, hubs or centres. ${ }^{8}$

Critical to the central focus of broadening the availability of global cardiac surgical services lies the foundational availability of training programmes and ancillary providers available to support basic cardiac services (ie, valve replacement) in a sustainable fashion. It is on this foundation that additional global cardiac surgical services may be expanded (ie, congenital and/ or paediatric cardiac surgery) to resource-constrained settings. With time, access to cardiac surgery has broadened; however, improved socioeconomic conditions in many of these developing economies have also witnessed major increases in the need for cardiac surgical services to address the continuing prevalence of rheumatic heart disease, the high incidence, prevalence and backlog of complex congenital heart disease, and the accelerated increase in coronary artery disease and degenerative valve and aortic diseases. As this clinical demand increases, the challenges in low-income and middle-income settings will continue to be access, cost, availability of services, demand for high-tech procedures, qualified healthcare personnel and the need for increased quality cardiac care centres. ${ }^{8}$

\section{CONCLUSION}

As we move towards the Sustainable Development Goals by 2030, addressing the unmet need of surgical care for cardiovascular diseases is paramount to reach the targets of reducing neonatal and under-five mortality, infectious diseases and non-communicable diseases. The cardiac surgery community can learn from the global neurosurgery community to establish a similar global movement woven into a multidisciplinary framework to address the global burden of cardiovascular disease and the gross inequities underlying access to cardiac surgical care. To do so, strengthening existing global frameworks and advocating for global cardiac surgery as professional societies and individuals is critical.

Contributors Conception of work: DV, KP. Drafting of article: DV, KP, JS. Final approval of the version to be published: DV, KP, JS.

Funding The authors have not declared a specific grant for this research from any funding agency in the public, commercial or not-for-profit sectors.

Competing interests None declared.

Patient consent for publication Not required.

Provenance and peer review Not commissioned; externally peer reviewed.

\section{REFERENCES}

1 Meara JG, Leather AJ, Hagander L, et al. Global Surgery 2030: evidence and solutions for achieving health, welfare, and economic development. Lancet 2015;386:569-624.

2 Reichert HA, Rath TE. Cardiac surgery in developing countries.J Extra Corpor Technol 2017:49:98-106.

3 Mock CN, Donkor P, Gawande A. Essential surgery: key messages from disease control priorities. . 3rd edn, 2015: 1. 1-11.

4 Park KB, Johnson WD, Dempsey RJ. Global neurosurgery: the unmet need. World Neurosurg 2016;88:32-5.

5 Dewan MC, Rattani A, Fieggen G, et al. Global neurosurgery: the current capacity and deficit in the provision of essential neurosurgical care. executive summary of the global neurosurgery initiative at the program in global surgery and social change. $J$ Neurosurg 2018:1-10.

6 Musa NL, Hjortdal V, Zheleva B, et al. The global burden of paediatric heart disease. Cardiol Young 2017;27-S3-S8.

7 Zilla P, Bolman RM, Yacoub MH, et al. The Cape Town declaration on access to cardiac surgery in the developing world. Eur J Cardiothorac Surg 2018;54:407-10.

8 Pezzella AT. Global cardiothoracic surgery advances and challenges in developing countries and emerging economies. Asian Cardiovasc Thorac Ann 2010:299-310. 